\title{
MME Positive
}

National Cancer Institute

\section{Source}

National Cancer Institute. MME Positive. NCI Thesaurus. Code C162087.

An indication that MME expression has been detected in a sample. 